\title{
Continuous noninvasive monitoring of cell growth in disposable bioreactors
}

\author{
T. Reinecke ${ }^{a, *}$, P. Biechele ${ }^{b}$, M. Sobocinski ${ }^{c}$, H. Suhr ${ }^{a}$, K. Bakes ${ }^{a}$, D. Solle ${ }^{b}$, H. Jantunen ${ }^{c}$, \\ T. Scheper ${ }^{b}$, S. Zimmermann ${ }^{a}$ \\ ${ }^{a}$ Institute of Electrical Engineering and Measurement Technology, Dept. of Sensors and Measurement Technology, Leibniz \\ Universität Hannover, Appelstr. 9A, 30167 Hannover, Germany \\ b Institute of Technical Chemistry, Leibniz Universität Hannover, Callinstr. 5, 30167 Hannover, Germany \\ c Microelectronics Research Unit, University of Oulu, Erkki Koiso-Kanttilan Katu 3, 90570 Oulu, Finland
}

\begin{abstract}
To ensure high quality output of biotechnological processes, relevant process parameters need to be monitored. As bioprocesses are increasingly executed in single use bioreactors, there is an increasing demand for new sensors applicable to these processes. In this work, we investigate different approaches for continuous non-invasive cell growth monitoring, especially for single use bioreactor applications. Therefore, the permittivity of the cell culture is used as a measure for the biomass. In a first step, a measuring procedure based on the transmission measurement of an electromagnetic wave is investigated. It appears that the penetration depth of this sensor is not sufficient for a noninvasive measurement through the polymer wall of a single use bioreactor. Therefore, alternative setups based on magnetic induction are investigated. The initial setup is very simple. It consists of a planar coil connected to an impedance analyzer. The coil is attached to the outside of the polymer foil of the single use bioreactor and an impedance spectrum is measured. To evaluate the sensor, E. coli cultivations are performed in a modified cultivation setup, which enables measurements through the polymer foil of a Sartorius BIOSTAT ${ }^{\circledR}$ CultiBag RM, and additionally allows sampling of culture medium for optical density reference measurements. The resonance peak of the coil in the impedance spectrum, is observed as measure for the optical density. Regardless of the simple sensor construction, we found a good correlation between optical density and the damping ratio of the resonance peak. However, the sensor signal shows saturation towards high optical densities. Therefore, an LTCC coil producing a higher magnetic flux density in the culture medium is investigated subsequently. This sensor shows a linear response up to high optical densities, but the sensitivity is reduced compared to the former used coil and therefore scattering of the data is increased. However, to increase the sensitivity, a linear variable differential transformer is realized. Using this setup, the influence of the primary magnetic flux is eliminated from the measuring voltage. This approach delivers the most promising results, as the sensor response is linear up to high optical densities and data scattering is low.
\end{abstract}

\section{Introduction}

Biotechnological processes become more and more important for the production of several products (e.g. food supplements, flavorings, recombinant proteins, antibodies, vaccines), especially in the fields of food and biopharmaceutical industries [1,2]. Most of these bioprocesses can be described as complex three-phase systems. The cells, as producers of the bioproducts, are dispersed as a solid phase in a liquid phase, the cell culture medium, which contains all necessary nutrients for the growth and survival of the cells. This liquid phase is aerated by the gas phase, for the supply of the cells with oxygen and the use of $\mathrm{CO} 2$ to maintain the $\mathrm{pH}$. The interactions between these three phases are complex. Biological components often react very sensitively to environmental changes (e.g. $\mathrm{pH}$, temperature, pO2, nutrients, toxic byproducts), which can lead to an impairment of the cell activity, productivity or the reproducibility of the process. For the control and optimization of cultivation processes for high product yields and quality, as well as for documentation purposes, a detailed analysis and monitoring of all parameters of these three phases in combination with broad process knowledge is necessary [3-6].

In order to better understand, observe and control these complex processes in biotechnological cultivation processes, it is necessary to work continuously on the development, improvement and 
optimization of existing and new sensor systems. This allows to get a better, comprehensive picture of the overall process and to initiate countermeasures in the event of fluctuations.

Another aspect of modern biotechnology is the use of single use bioreactors (SUBs), instead of classical stainless steel reactors. These disposable reactor systems offer some advantages, the most important are the increased facility flexibility, lower investment, cleaning and energy costs and especially a lower risk of contaminations, as these disposables are pre-sterilized by gamma radiation [7].

The continuous measurement of biomass is one of the most important parameters, which have to be determined. It gives an overview over the process performance, the process state and condition of the used cells. Common, classical sensors for this purpose are not directly usable in disposable systems due to their set-up, as they cannot be inserted in the pre-sterilized disposable SUB, or external off - line measurement has to be performed. There are some other possibilities [8-14] to implement modern sensor systems at bioreactor systems, also some that can endure gamma-radiation in the SUB, before the whole SUB gets sterilized by the manufacturer, but this is very expensive and elaborate.

To overcome this issues, we explore different non-invasive approaches for monitoring cultivation process parameters through the SUB polymer foil. Hereby, the sensor is attached at the outer site of the SUB and the measurement is performed through the foil. This enables one to measure process parameters noninvasively, without any contact to the culture broth, which reduces the risk of contaminations and allows implementing sensors independently of the manufacturer of the SUB.

All approaches for noninvasive cell growth monitoring presented in this work are based on a complex permittivity measurement of the cell culture. However commercially available capacitive sensors for dielectric spectroscopy, like the ones used in [15-17] are not suitable for noninvasive monitoring of the cell density through the polymer wall of a SUB. This can be visualized via an electromagnetic simulation with CST Microwave Studio.

Fig. 1 (left) shows a measurement with a capacitive probe in direct contact with the culture medium. The electrical field has a high penetration depth into the culture medium and accurate determination of the cell density is possible. However, when the capacitive probe is attached to the outside of a SUB, as shown in Fig. 1 (right), there is a high field strength in the polymer foil and therefore the penetration depth into the medium is decreased, which is attributed to the low permittivity $\left(\varepsilon_{r}=2-\varepsilon_{r}=4\right)$ of the polymer foil. Since the medium is virtually field free, cells cannot be polarized, which would be necessary for a capacitive cell growth measurement.
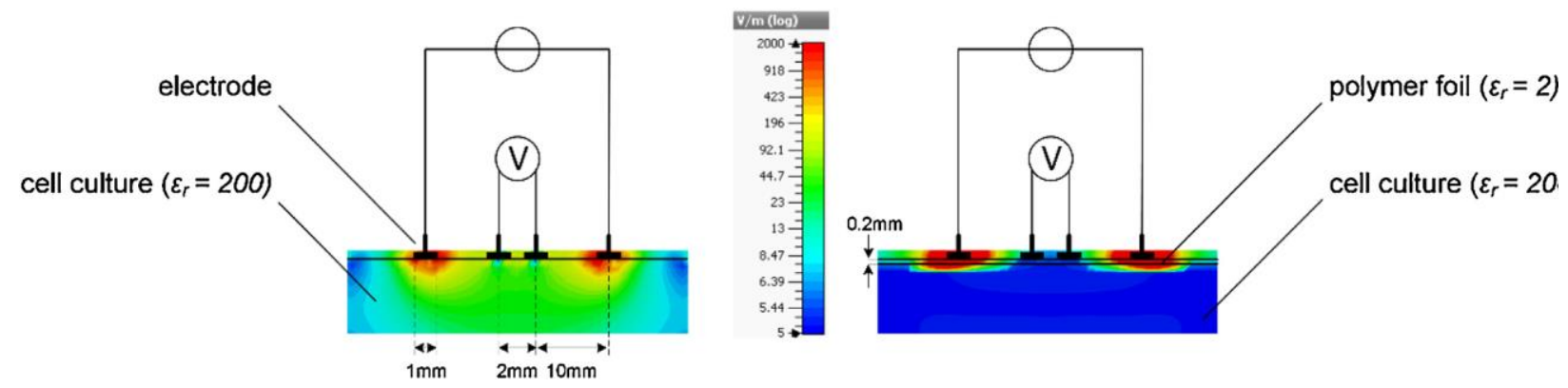

Fig. 1. Four-electrode measurement without (left) and with (right) polymer foil (CST Simulation at $1 \mathrm{kHz}$ with discrete port excitation (1 V Amplitude), 190000 Hexahedral Mesh cells and open (add space) boundaries).

High frequency microwave sensors showed some promising results for noninvasive cell growth monitoring as presented in $[18,19]$. Therefore, we also investigated a procedure for monitoring cell growth via a transmission measurement of an electromagnetic wave in [20]. This technique employed for permittivity measurements is known to be very sensitive [21]. The used sensor in this work is the coplanar transmission line depicted in Fig. 2(a). It was originally designed for complex permittivity measurements of tissue in the $\mathrm{MHz}$ regime in order to determine the water content [22]. Based on the successful employment of this sensing principle, it was subsequently transferred to cell growth monitoring at low frequencies.

The sensor structure consists of an inner conductor centered between two ground planes, all assembled on a RO6010 substrate with a relative permittivity of $\varepsilon_{\mathrm{r}}=10.2$ (at $10 \mathrm{GHz}$ and $23^{\circ} \mathrm{C}$ ) and $2.54 \mathrm{~mm}$ 
thickness. The copper cladding has a thickness of $35 \mu \mathrm{m}$, whereby the backside metallization was removed. The space between inner conductor and ground plane is $s=1.1 \mathrm{~mm}$ and the width of the inner conductor is $\mathrm{w}=2.8 \mathrm{~mm}$, leading to a matched impedance of $Z_{0}=50 \Omega$ for the unloaded transmission line in air $\left(\varepsilon_{\mathrm{r}}=1\right)$ at high frequencies. Note that a possible change in substrate permittivity low frequencies $(\mathrm{kHz})$ was not accounted for. With an edge length of $\mathrm{I}=20 \mathrm{~mm}$, the size of the quadratic sensing area is $400 \mathrm{~mm}^{2}$.

For a preliminary investigation of the sensors' general ability to monitor cell growth, it is submerged in the culture medium, while the cultivation process is performed in a flask. For all experiments shown in this work, cells from an over-night pre culture were inoculated with an OD of 0.1. As cultivation media, we used classical LB media, consisting of $5 \mathrm{~g} / \mathrm{L}$ yeast extract, $10 \mathrm{~g} / \mathrm{L}$ sodium chloride, $5 \mathrm{~g} / \mathrm{L}$ tryptone and $0.5 \mathrm{~g} / \mathrm{L} \mathrm{glucose}$. To prevent agglomeration of cells, the culture is placed on a $25 \mathrm{~mm}$ orbital shaker at $180 \mathrm{rpm}$.
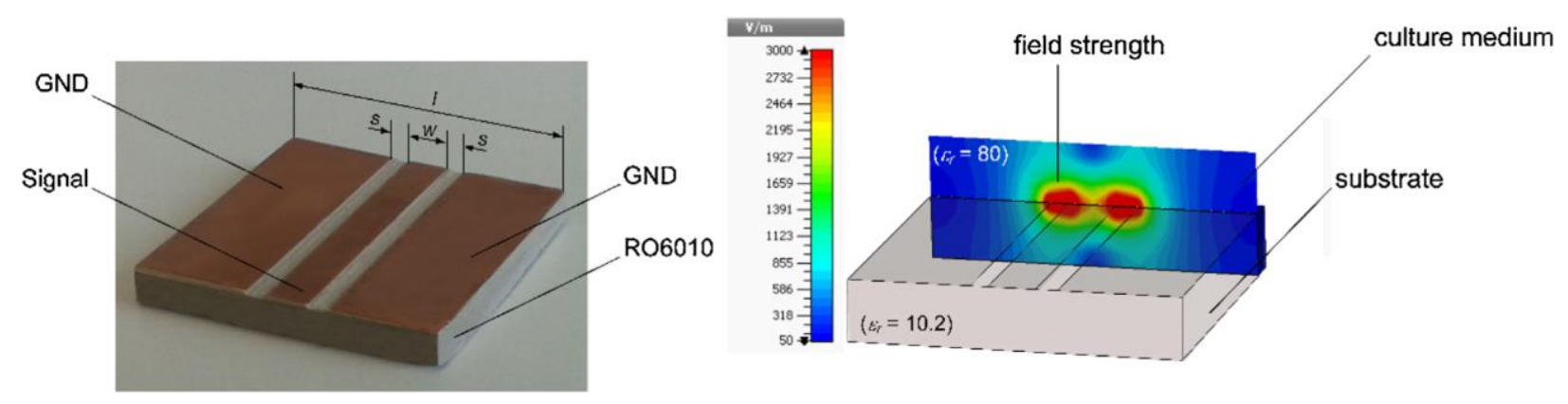

Fig. 2. (a) Photo of a coplanar transmission line, (b) CST simulation of the electrical field (CST Simulation at $1 \mathrm{kHz}$ with waveguide port excitation, 11000 Hexahedral Mesh cells and $\mathrm{H}_{\mathrm{t}}=0$ boundary condition).

Fig. 2(b) depicts a CST Microwave Studio simulation of the coplanar transmission line submerged in the cell culture. Here, the electromagnetic field is partly inside the substrate and partly inside the culture medium above the transmission line. Thus, the propagation of an electromagnetic wave through the coplanar transmission line is influenced by the permittivity of the culture medium and therefore by the cell density in the medium. To find an optimum measurement frequency, we performed measurements of the effective permittivity (the effective permittivity is a superposition of the substrate permittivity and the permittivity of the cell culture) in the frequency range between $1 \mathrm{kHz}$ and $1 \mathrm{GHz}$ using the algorithm described in $[23,24]$ to calculate the effective Permittivity from the measured forward transmission (scattering parameter $S_{21}$ ). It was found that the best results for the determination of the OD from the measured effective permittivity are achieved at a frequency of $1 \mathrm{kHz}$, although at this low frequency, the $20 \mathrm{~mm}$ long coplanar transmission line is small compared to the several kilometer long wavelength. However, cells exhibit high permittivity values at $\mathrm{kHz}$-frequencies $\left(\varepsilon_{\mathrm{r}} \approx 10^{6}[25]\right)$, significantly reducing the wavelength, which is an explanation for the determined frequency optimum.

At the measurement frequency of $1 \mathrm{kHz}$, we found a linear correlation with a coefficient of determination $R^{2}=0.98$ between the real part of the effective permittivity and the optical density at $600 \mathrm{~nm}$, measured with a Multiskan GO, as depicted in Fig. 3. Here, the effective permittivity is normalized to the measured effective permittivity of the culture medium before the beginning of cell growth in order to eliminate the influence of temperature differences between single cell cultivations or change in substrate permittivity. The results proof that cell growth monitoring is generally possible with our setup, but the goal of this work is monitoring cell growth non-invasively through the polymer wall of a SUB. Therefore, the sensor is attached to the foil of a Satorius BIOSTAT Cultibag RM. 


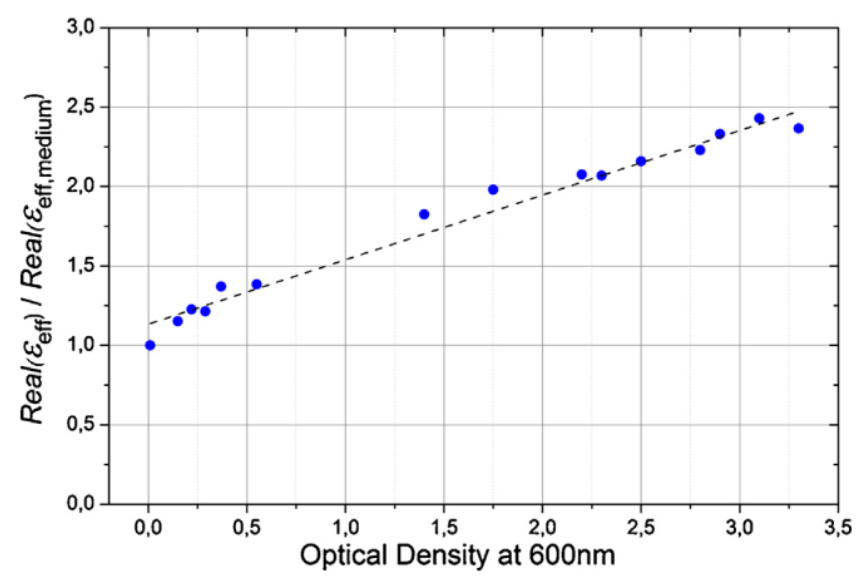

Fig. 3. Linear dependency between real part of the effective permittivity and optical density.

The corresponding CST Simulation in Fig. 4 shows that the sensor suffers from the same Problem as all the capacitive sensors: The field is concentrated in the polymer foil and thus the electromagnetic field in the culture medium is decreased, resulting in virtually no sensor response when measuring through the foil.

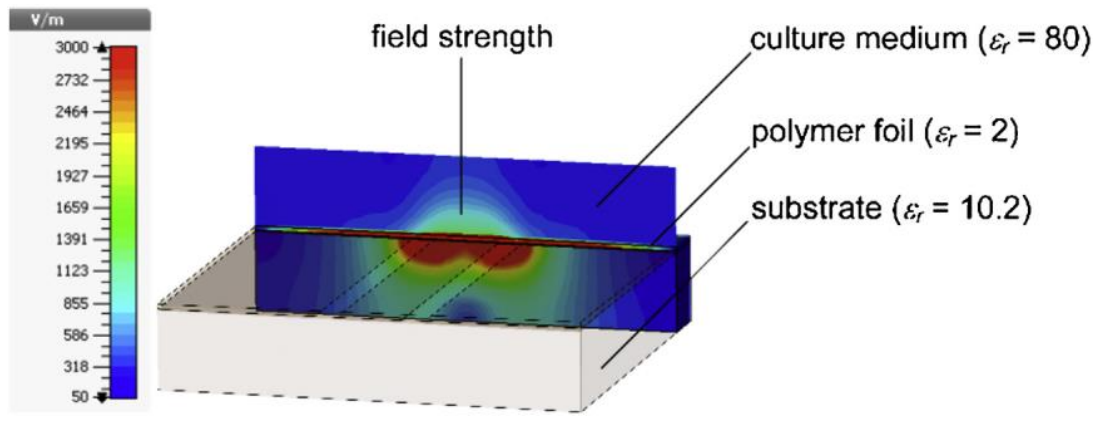

Fig. 4. CST simulation of the electrical field for a sensor attached to the SUB (CST Simulation at $1 \mathrm{kHz}$ with waveguide port excitation, 11000 Hexahedral Mesh cells and $\mathrm{H}_{\mathrm{t}}=0$ boundary condition).

Subsequently, we attempted to improve the sensor performance by increasing the penetration depth. Therefore, a miss-matched sensor is realized by increasing the spacing between inner conductor and ground plane to $s=7.15 \mathrm{~mm}$. Furthermore, the width of the inner conductor is decreased to $w=2 \mathrm{~mm}$. With this sensor, we could show that it is generally possible to monitor the cell density noninvasively from outside the SUB using a coplanar transmission line, but it appeared that the sensor is very sensitive to disturbances and thus accuracy and reproducibility are limited and not entirely satisfactory.

The aim of this paper is a preliminary investigation of different approaches, which could lead to the realization of a measuring system, capable of determining relevant biotechnological process parameters noninvasively through the polymer foil of a SUB.

\section{Noninvasive inductive cell growth monitoring}

It was shown via simulations and measurements that capacitive coupling between sensor and culture medium is not a promising approach for measuring cell density noninvasively through the polymer wall of a SUB. However, as the foil does not disturb the magnetic flux density, a measurement procedure based on magnetic induction is investigated in this section.

Fig. 5 depicts a schematic of the measuring principle for non-invasive cell growth monitoring presented in [26]. The corresponding setup is very simple: it only consists of an impedance analyzer and a two-sided planar coil with a diameter of $34 \mathrm{~mm}$ on a standard FR4 substrate. The inductivity of the coil is approx. $L=30$ $\mu \mathrm{H}$. The general idea is to simply attach the coil to the outside of the polymer foil of a SUB. When a current flows through the coil, the primary magnetic flux induces an electrical field in the culture medium. This 
electrical field subsequently produces currents in the medium: First, eddy currents depending on the electrical conductivity, and second displacement currents depending on the relative permittivity of the cell culture. These currents produce a secondary magnetic flux in the culture medium. The measuring effect is the cell density dependent feedback effect of the secondary magnetic flux on the primary magnetic flux of the coil. The basic idea is similar to the investigation of inductive cell growth monitoring in $[27,28]$. Here, a commercially available inductive probe (HP E5050A Colloid Dielectric Probe, Hewlett-Packard) is used for dielectric measurements. The probe consists of two magnetically coupled coils for application in traditional bioreactors. However, it is mandatory to submerge the probe into the culture medium, as the medium has to be located between the coils and therefore it is not applicable to SUBs.

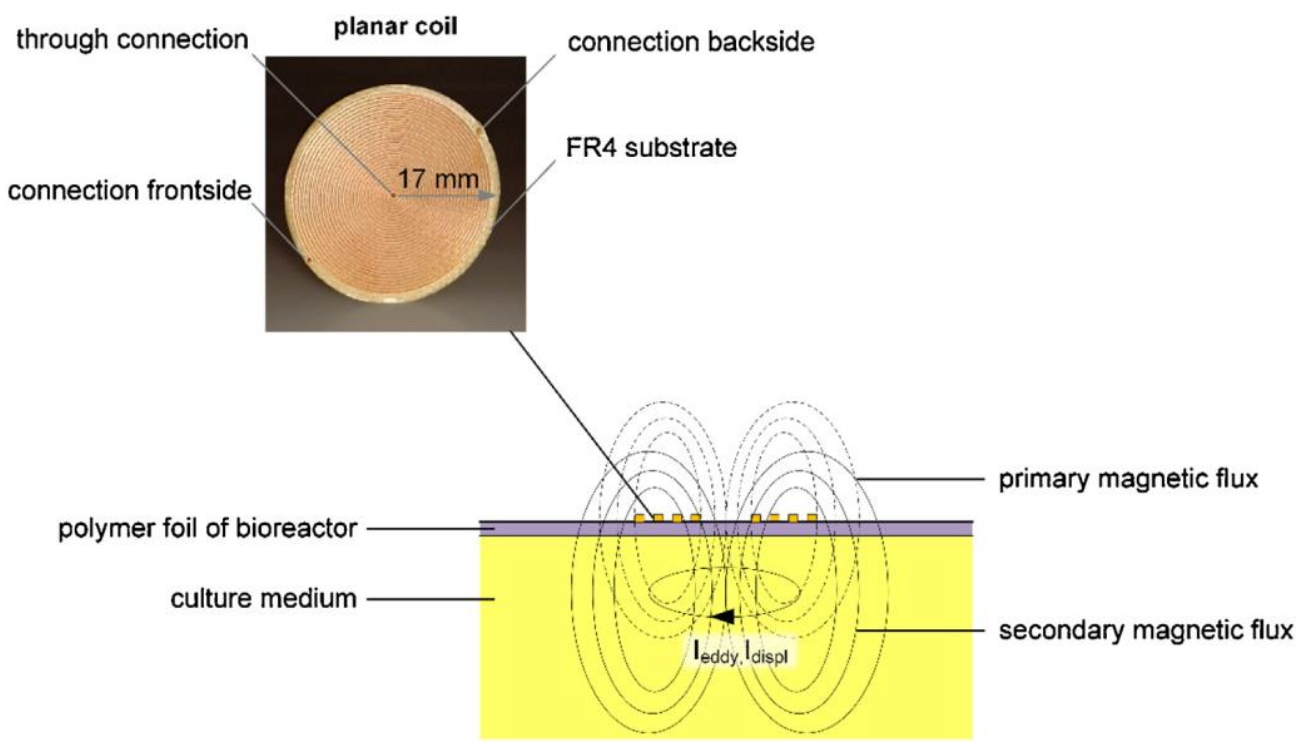

Fig. 5. Schematic of the measuring principle for noninvasive inductive cell growth monitoring.

In Fig. 6, impedance spectra of the planar coil attached to the SUB polymer foil of a Sartorius BIOSTAT ${ }^{\circledR}$ CultiBag RM are shown. All spectrums are averaged 16 times, leading to a measuring time of approximately $30 \mathrm{~s}$ per spectrum. The straight line is the measured spectrum when the foil is not in contact with culture medium. Combined with the intrinsic capacitance $C_{p}$, the coil behaves like a parallel resonant circuit, with a resonance frequency of $f_{\text {res }}=1 /\left(2 \pi \sqrt{L C_{p}}\right) \sqrt{1-D^{2}}=11.47 \mathrm{MHz}$. With the $3 \mathrm{~dB}$ bandwidth $\mathrm{BW}_{3 \mathrm{~dB}}$, the damping ratio of the unloaded resonator can be determined to $D=B W_{3 \mathrm{~dB}} / 2 \mathrm{f}_{\text {res }}=0.019$. However, when the

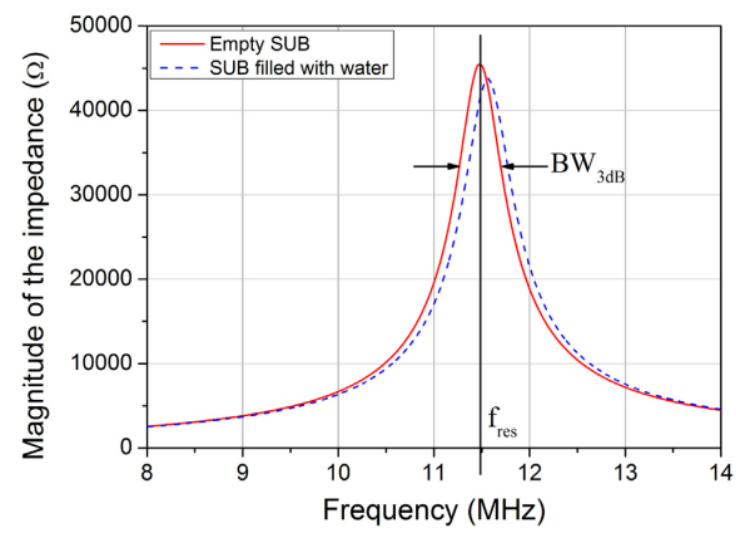

Fig. 6. Impedance spectrum of the unloaded planar coil.

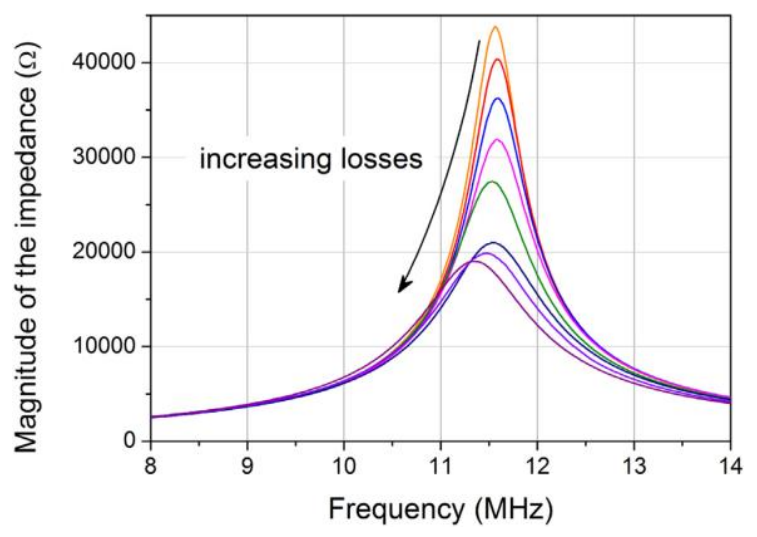

Fig. 7. Impedance spectra of a measurement of saline with increasing $\mathrm{NaCl}$ concentration from $0 \mathrm{~g} / \mathrm{ml}$ to $0.03 \mathrm{~g} / \mathrm{ml}$.

bioreactor is filled with DI-Water, the resonance frequency shifts towards a higher frequency, indicating a decreased inductivity $L$ and therefore an inductive measuring effect. Because if the measuring effect would 
be capacitive, increasing the permittivity of the medium from $\varepsilon_{\text {Air }}=1$ to $\varepsilon_{\text {Water }}=80-\mathrm{j} 2$ [29] would result in a lower resonance frequency, as the intrinsic capacitance would increase. However, the setup is also sensitive to parasitic fluctuations of capacitance, e.g. brought by movement of connecting cables or electromagnetic coupling into connecting cables. Therefore, we used shielded coaxial lines with fixed position to minimize the influence of disturbances."

In the next step, the sensor response is investigated via measurements of saline to model the presence of a culture medium. The measured impedance spectra are depicted in Fig. 7. Increasing the $\mathrm{NaCl}$ concentration leads to an increase of losses, as conductivity and polarization losses of the aqueous solution are increasing [29]. It can be seen that the senor is sensitive to losses in the medium in contact with the polymer foil: The amplitude of the resonance Peak lowers and the bandwidth increases when increasing $\mathrm{NaCl}$ concentration. Calculating the damping ratio $D$ shows a linear dependency between $D$ and the $\mathrm{NaCl}$ concentration.

Subsequently, we performed $E$. coli cell cultivations in our experimental setup, which allows both measurements through the polymer foil of the SUB, and sampling of the cell culture for optical density (OD) reference measurements. Fig. 8(a) depicts the bacterial growth curve of $E$. coli determined with the Multiskan $\mathrm{GO}$ at a wavelength of $600 \mathrm{~nm}$ at equidistant $30 \mathrm{~min}$ time steps. The growth curve shows an exponential behavior and cell density almost doubles in the interval of $30 \mathrm{~min}$. After $210 \mathrm{~min}$ growth time, the biomass reaches its maximum with an OD of 3.7. In parallel to the OD determination, non-invasive impedance measurements are performed. With a resonance frequency in the range of $f_{\text {res }}=11 \mathrm{MHz}-12 \mathrm{MHz}$, the measuring frequency is too high to expect an influence of cell polarization, as the frequency is beyond the beta-dispersion of the real part of the permittivity, as described in [25]. However, increasing biomass leads to increasing dielectric losses [30], which can be detected even at high frequencies. Therefore, the damping ratio increases with increasing cell density, analog to the measurements with saline in Fig. 7.

Fig. 8(b) shows the dependency of the damping ratio from the OD. At low optical densities, a linear dependency between OD and D can be assumed. Towards higher ODs, a saturation of the sensor signal can be observed. However, this simple sensor enables monitoring of cell growth up to an optical density of OD $\approx$ $2-3$.
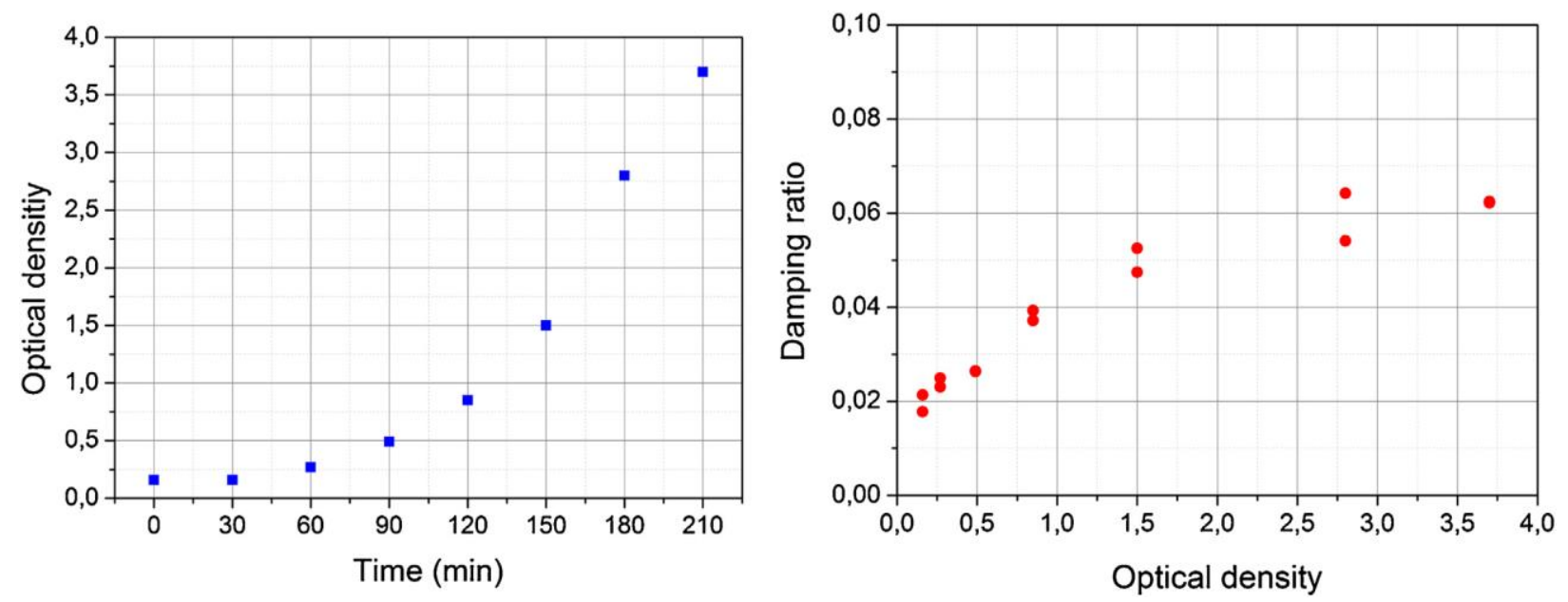

Fig. 8. (a) Bacterial growth curve of cell culture; (b) Damping ratio in dependence of the optical density measured at $600 \mathrm{~nm}$.

To increase the dynamic range, Low Temperature Co-fired Ceramic (LTCC) coils having a higher magnetic flux density were subsequently tested. LTCC technology is commonly used in modern day electronics. LTCC systems consist of dielectric ceramic tapes and functional pastes which together form multilayer structures than can be sintered in one process (co-fired) at a relatively low temperature of $850{ }^{\circ} \mathrm{C}$. Co-firing and a variety of functional pastes allows creating complex structures with embedded passive components (resistors, capacitors, inductors). Integrated passives and multilayer structures make LTCC a perfect technology for smart packages, RF devices, sensors and even microfluidics [31,32]. 
Typical LTCC process includes blanking, via forming, via filling, screen printing, stacking, laminating and co-firing followed by any necessary post-processing such as dicing and surface mounting [31,32].

LTCC inductors have been under careful study for some time [33-36]. The basic design consists of one or more planar spirals connected together with metalized vias.

The coils used in this paper were manufactured using a DuPont 951 LTCC system. Two mirrored designs were prepared in order to assure current flow in single direction. Designs were transferred to screen-printing stencils with 325 mesh and $16 \mu \mathrm{m}$ thick emulsion. LTCC tapes with thickness of $150 \mu \mathrm{m}$ were blanked to fit our process line and have connection vias formed by laser cutting. The vias were filled with DuPont 6141 silver via fill and dried in a box oven for $10 \mathrm{~min}$ at $100^{\circ} \mathrm{C}$. In the next step, coil patterns were screen-printed using DuPont 6142 silver inner conductor and a semi-automated screen printer. Subsequently, the layers were stacked on an aligning table where register pins assured good enough alignment between the layers. Stacking is performed in a manner that odd layers had right-winded coils and even layers had left-winded coils. The tapes were then laminated in an isostatic laminator at $75{ }^{\circ} \mathrm{C}$ for $10 \mathrm{~min}$ at $20 \mathrm{MPa}$. Finally, the structures were co-fired in a box furnace according to vendor's guidelines and diced into singular pieces, shown in Fig. 9. The coil has a diameter of $d=7.5 \mathrm{~mm}$ and an inductivity of approx. $5 \mu \mathrm{H}$.
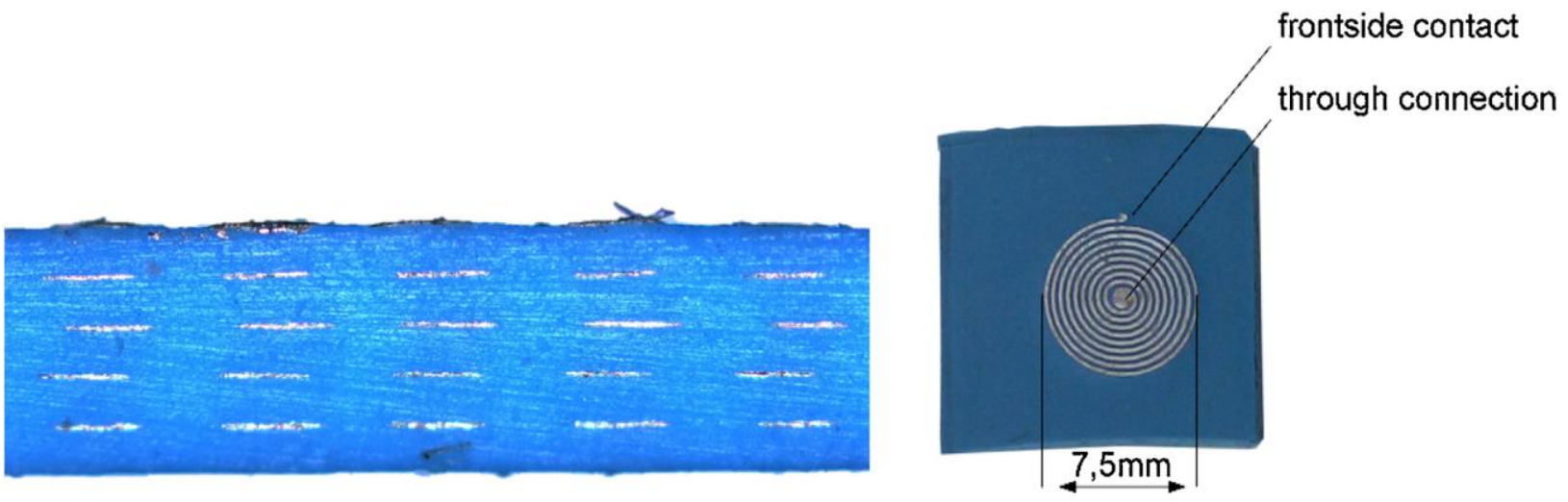

Fig. 9. Cross-section through (a) and top-view of the coil (b).

Analog to the characterization of the PCB coils, measurements of saline were performed with the LTCC coils to investigate the sensor response to losses in the medium. As the resonance frequency of the coil is above the maximum measuring frequency of the employed impedance analyzer of $20 \mathrm{MHz}$, we use two coils connected in series and additionally a small capacitance of $C=6.8 \mathrm{pF}$ is connected in parallel to shift the resonance frequency of the unloaded resonance circuit to approx. $17.5 \mathrm{MHz}$, as shown in Fig. 10.

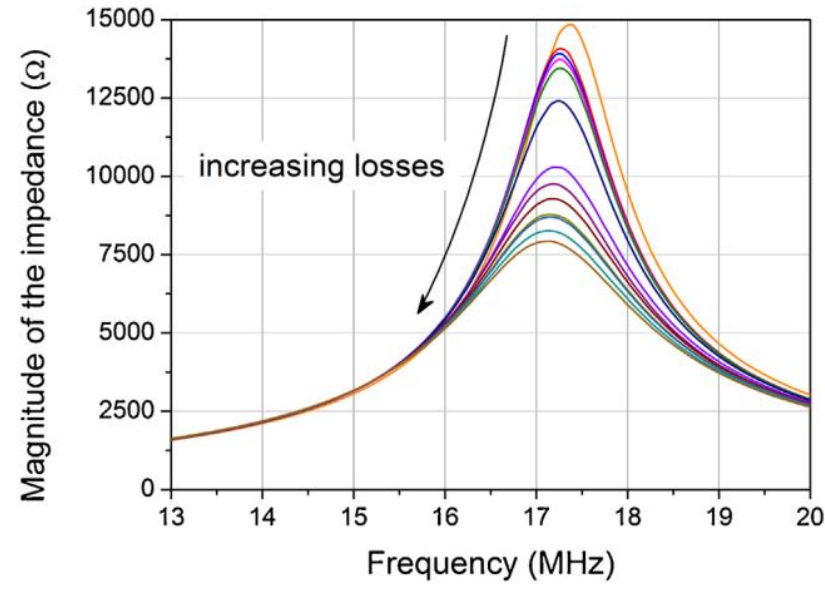

Fig. 10. Characterization of the LTCC coil via measurements of saline through the bioreactor foil with increasing $\mathrm{NaCl}$ concentration from 0 $\mathrm{g} / \mathrm{ml}$ to $0.03 \mathrm{~g} / \mathrm{ml}$.

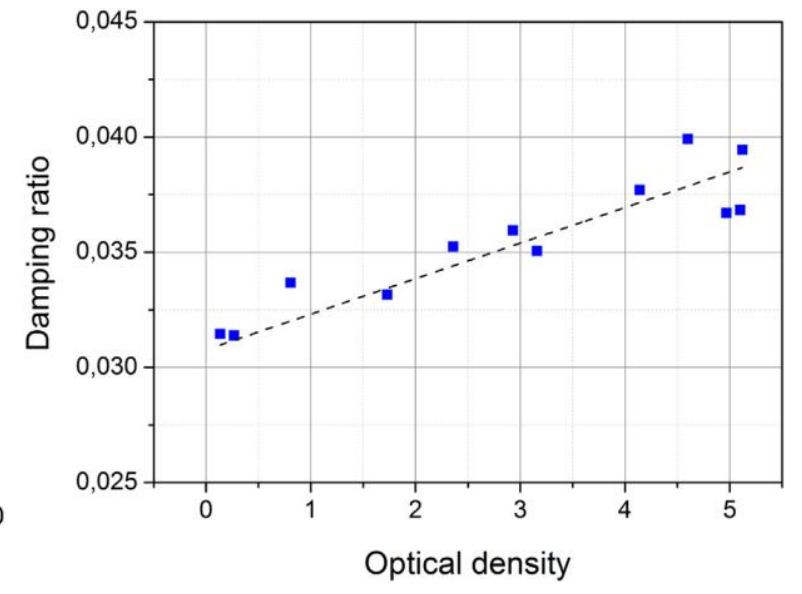

Fig. 11. Damping ratio of the LTCC coil versus optical density in the cell culture. 
Similar to the response of the PCB coil to increasing losses in the medium, the LTCC coil shows an increasing damping ratio towards higher $\mathrm{NaCl}$ concentrations. Subsequently the cell cultivation process of $E$. coli is monitored with the LTCC coil. The results are depicted in Fig. 11. In contrast to the PCB coil, it can be seen that there is no saturation of the sensor signal, up to an optical density of $O D=5$. A simple explanation is the reduced sensitivity of the LTCC coils: Comparing the dependency between damping ratio and optical density of the LTCC coil in Fig. 11 to that of the PCB coil in Fig. 8(b) reveals that the slope of the linear section in Fig. $8(\mathrm{~b})$ is considerably higher for the PCB coil. Therefore, the LTCC coils have a higher dynamic range and saturation occurs at higher optical densities compared to the measurement with the PCB coil. However, due to the reduced sensitivity, the data points in Fig. 11 show a higher scattering, resulting in a poor coefficient of determination $\mathrm{R}^{2}=0.82$.

Thus, one approach for increasing sensitivity using LTCC coils could be the use of more than two coils connected in series in order to increase the sensing area. In this way, an optimum could be found between sensitivity and OD-range that fits the application. However, in the next section we investigate the employment of a differential setup in order to increase sensitivity without losing dynamic range.

\section{Linear variable differential transformer for cell growth monitoring}

The limiting factor when using only a single coil is that the measuring signal is dominated by the influence of the primary flux of the coil and thus the weak secondary flux from the medium is hardly detectable. Therefore, the employment of a linear variable differential transformer (LVDT) for noninvasive cell growth monitoring is investigated in this section. The LVDT is a differential setup, where the influence of the primary flux through the measuring coil is eliminated from the measuring voltage. LVDTs are widely used, e.g. as a displacement sensor for high precision measurement of plant growth [37], venous compliance for assessment of drug effects [38] or bioadhesion to cervical tissue [39]. A schematic of the setup employed in this work is depicted in Fig. 12. It consists of three coils, all assembled on a ferrite core $\left(\varepsilon_{r}=300\right)$. The coils are wound with an insulated copper wire with a diameter of $1.8 \mathrm{~mm}$. All three coils have a similar inductance

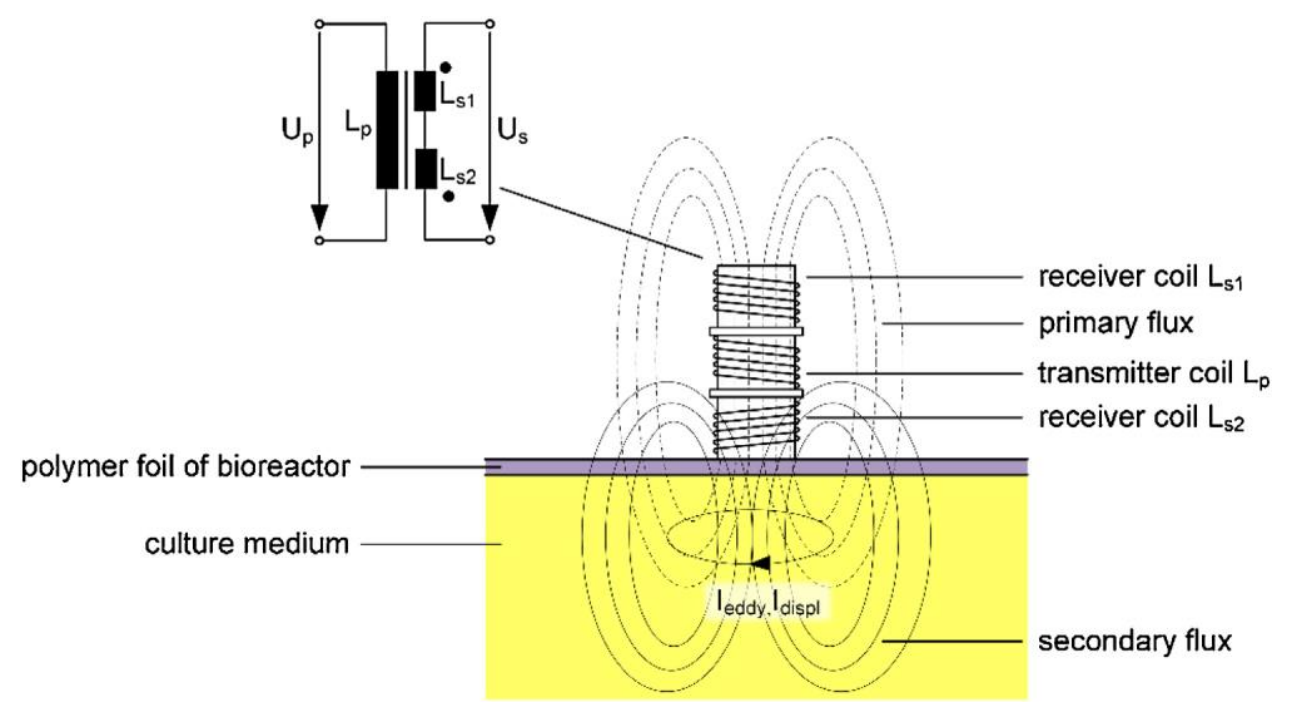

Fig. 12. Setup with linear variable differential transformer.

of approximately $L=1 \mathrm{mH}$ and a resonance frequency between $500 \mathrm{kHz}$ and $600 \mathrm{kHz}$. The center coil $L_{p}$ produces an identical flux through the receiver coils $L_{s 1}$ and $L_{s 2}$. As the windings of $L_{s 1}$ and $L_{s 2}$ are in opposite directions, the induced voltages have an opposite sign and thus cancel out from the measuring Voltage $U_{s}$. Therefore, the measuring voltage is $U_{s}=0$ for an unloaded sensor. To achieve a maximum compensation of the flux, the coil $L_{s 1}$ can be adjusted in height, allowing fine-tuning. When the sensor is attached to the SUB, the primary flux induces eddy and displacement currents in the medium, analog to the procedure described in the previous section. The secondary flux from these currents only induces a voltage in $L_{s 2}$ as it is placed much closer to the medium than $L_{s 1}$. Therefore, the measuring voltage $U_{s}$ equals the induced voltage in $L_{s 2}$. 
The interaction between primary flux and medium as well as the effect on the measuring voltage $U_{s}$ can be described by the equivalent circuit shown in Fig. 13.

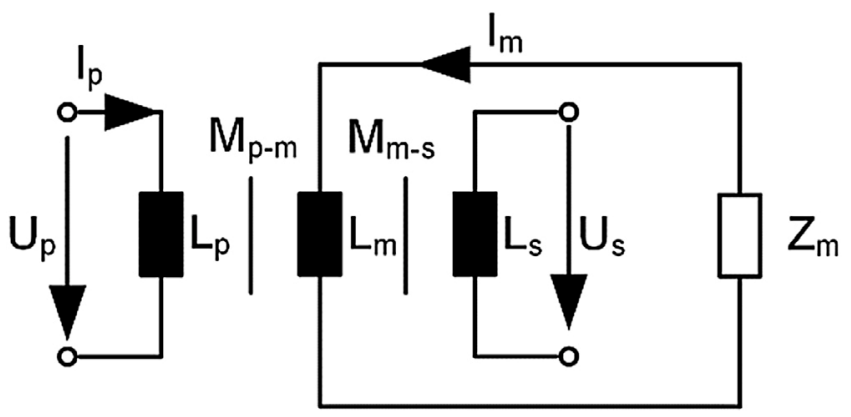

Fig. 13. Equivalent circuit of the linear variable differential transformer coupled to the culture medium.

Here, $M_{p-m}$ is the mutual inductance between primary coil $L_{p}$ and the medium. $L_{m}$ is the inductance of the medium and $Z_{m}$ is an impedance representing conductive and capacitive effects of the medium. It is important to note that even without conductivity, displacement current are present in the medium producing a secondary magnetic field. $M_{m-s}$ is the mutual inductance between secondary coil $L_{s}$ and the medium. Note that the mutual inductance for the coupling between $L_{p}$ and $L_{s}$ does not need to be considered, as the primary flux through $L_{s 2}$ is compensated by $L_{s 1}$, as described before. Kirchhoff's loop for the primary coil yields

$$
U_{p}=j \omega L_{p} I_{p}+j \omega M_{p-m} I_{m} \stackrel{I_{m} \ll I_{p}}{\longrightarrow} U_{p}=j \omega L_{p} I_{p}
$$

To get more handy terms, it is assumed that the current in the medium is much smaller than the current through $L_{p}$, although this idealization is not necessary, as a feedback effect of $I_{m}$ on $I_{p}$ would be canceled out by the differential setup. Kirchhoff's loop for the medium yields

$$
I_{m} Z_{m}=-j \omega L_{m} I_{m}-j \omega M_{p-m} I_{p} .
$$

With Eqs. (1) and (2), the current in the medium can be calculated to

$$
I_{m}=\frac{-M_{p-m}}{L_{p}\left(Z_{m}+j \omega L_{m}\right)} U_{p} \stackrel{j \omega L_{m} \ll Z_{m}}{\longrightarrow} I_{m}=\frac{-M_{p-m}}{L_{p} Z_{m}} U_{p}
$$

It was experimentally verified via measurements of saline that the assumption of a negligible selfinductance of the medium $L_{m}$ is valid for low conducting materials like the culture medium. Even at comparably high $\mathrm{NaCl}$ concentrations of $5 \%$, no influence of the self-inductance could be detected. However, note that the influence of $\omega \mathrm{L}_{\mathrm{m}}$ becomes dominant for highly conducting materials (e.g. metals) at high frequencies.

With Kirchhoff's loop for the secondary coil, the measuring voltage in dependence of the current in the medium is

$$
U_{s}=j \omega M_{m-s} I_{m}
$$

Together with Eq. (3), the measuring voltage can be expressed in dependence of the primary voltage and the impedance of the culture medium as

$$
U_{s}=-j \omega M_{m-s} \frac{M_{p-m}}{L_{p}} U_{p} \frac{1}{Z_{m}} .
$$

The culture medium is modeled as a parallel circuit of a capacitance $C \sim \varepsilon_{r \text {,medium }}=\varepsilon_{r}^{\prime}+\varepsilon_{r}^{\prime \prime}$ and a conductance $\mathrm{G} \sim \kappa_{\text {medium }}$ and thus

$$
\frac{1}{z_{m}}=j \omega C+G
$$

With Eq. (6), the measuring voltage can be expressed in dependence of the complex permittivity of the medium $\varepsilon_{r \text {,medium }}$ and the conductivity $\kappa_{\text {medium }}$ as 


$$
U_{s}=\omega^{2} M_{m-s} \frac{M_{p-m}}{L_{p}} U_{p} \cdot C-j \omega M_{m-s} \frac{M_{p-m}}{L_{p}} U_{p} \cdot G=K_{1} U_{p} \varepsilon_{r}^{\prime}-j K_{2} U_{p}\left(\omega \varepsilon_{r}^{\prime \prime}+\kappa\right) .
$$

It can be seen that $U_{s}$ has a component which is in-phase to $U_{p}$, depending on the polarizability of the medium $\left(\varepsilon_{r}{ }^{\prime}\right)$ and a quadrature component depending on the dielectric losses $\left(\varepsilon_{r}{ }^{\prime \prime}\right)$ and the conductivity $(\kappa)$. To separate both influences, $U_{s}$ is interpreted as a complex signal and demodulated via IQ-demodulation. Then the in-phase component is

$$
U_{s}\left(\varepsilon_{r}^{\prime}\right)=\operatorname{Mean}\left(U_{s} \cdot \frac{U_{p}}{\widehat{U}_{p}}\right)
$$

Analog, the quadrature component can be derived via multiplication of the measuring voltage with the phase-shifted primary voltage to

$$
U_{s}\left(\varepsilon_{r}^{\prime \prime}, \kappa\right)=\operatorname{Mean}\left(U_{s} \cdot \frac{U_{p}}{\widehat{U}_{p}} e^{j 90^{\circ}}\right) .
$$

Besides an increase of sensitivity due to the compensation of the primary flux, a major advantage of this setup compared to the previously described setups is that the measuring frequency is not limited to the resonance frequency of the coil. The LVDT allows measurements of conductivity and permittivity in the frequency range of a few $100 \mathrm{~Hz}$ up to the resonance frequency of the coils at about $500 \mathrm{kHz}$. In a first step, the sensor is investigated via measurements of water isopropanol mixtures. The corresponding theoretical permittivity values were calculated using [40]. The measuring frequency is set to $300 \mathrm{kHz}$. Every measuring point is averaged 16 times, leading to a measuring time of approximately $20 \mathrm{~s}$.

As predicted by Eq. (8), Fig. 14 shows a linear dependency between the real part of the measuring voltage Us' and the polarizability of the medium $\left(\varepsilon_{r}^{\prime}\right)$ with a coefficient of determination $R^{2}=0.99$.

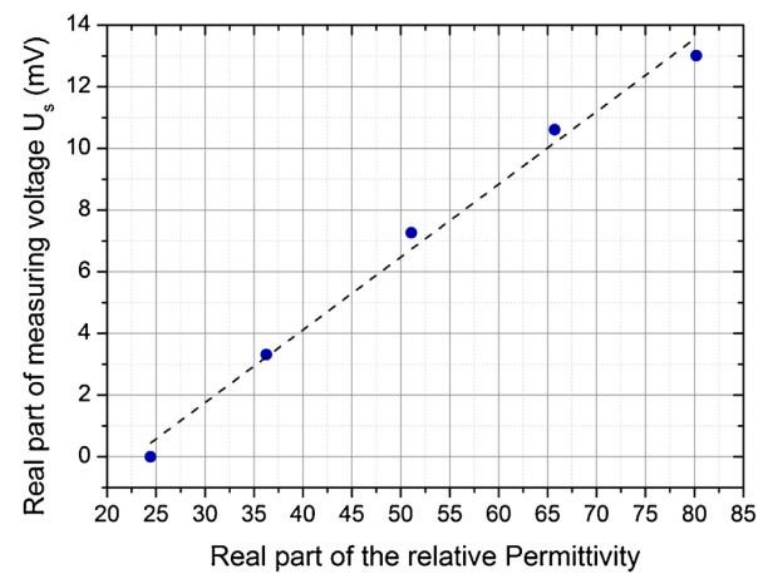

Fig. 14. Characterization of the differential setup with water isopropanol mixtures.

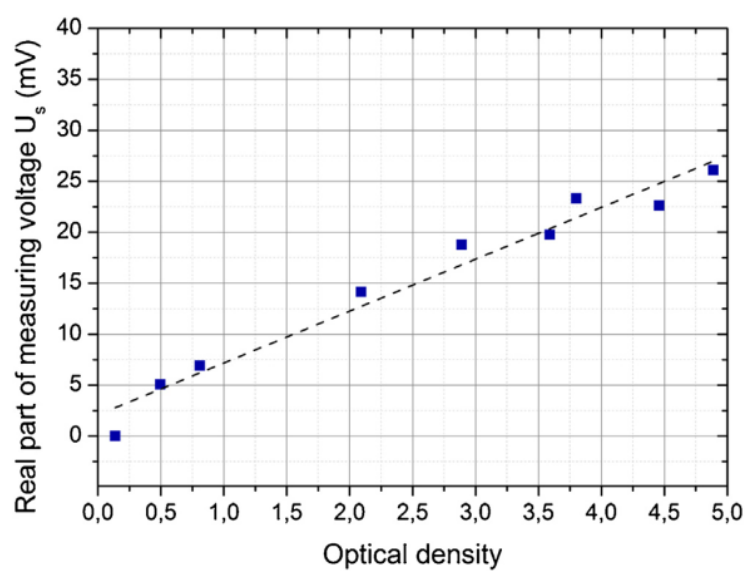

Fig. 15. Real part of the output voltage versus optical density.

Subsequently, we perform cultivation of $E$. coli and measure the cell density through the polymer foil with the LVDT and in parallel the OD with the Multiskan Go as reference. As the measuring frequency of $300 \mathrm{kHz}$ is considerably lower than the resonance frequency of the single coil approaches, cell polarization can follow the alternating electrical field. This leads to a huge influence of the cell density on the real part of the relative permittivity of the culture medium [25]. Fig. 15 shows a linear dependency with a coefficient of determination $\mathrm{R}^{2}=0.98$ between $U \mathrm{~s}^{\prime}\left(\varepsilon_{r}^{\prime}\right)$ and the optical density of the culture medium up to an optical density of $O D=5$. The scattering of the data from this preliminary experiment is small compared to the results achieved with the LTCC-coils and therefore we conclude that the LVDT is the most promising approach for non-invasive continuous cell growth monitoring through the polymer wall of SUBs.

\section{Conclusion}

In this work opportunities for simple continuous noninvasive monitoring of cell growth in disposable bioreactors where investigated to find an approach that could lead to the realization of an adequate measuring system. Via simulations and measurements with a sensor based on a coplanar transmission line, 
it could be shown that a capacitive coupling between sensor and culture medium leads to significantly decreased penetration depth of the sensor when measuring through the polymer wall of a SUB. Therefore, these sensors are not promising for this dedicated purpose, as they lack of sensitivity. However, as the magnetic flux can penetrate through materials with low permittivity and conductivity, and that virtually without any losses, we investigated setups based on magnetic induction. The first approach is very simple: A planar coil is attached to the outside of a SUB and the feedback effect of the secondary magnetic flux originating from the induced currents in the medium is observed via changes of the damping ratio of the resonance peak of the coil. As the sensor response from the primarily used PCB coil showed saturation towards high optical densities, a highly inductive LTCC coil was used subsequently. Here, the sensor response is linear up to optical densities of $O D=5$. However, as the sensitivity is reduced compared to the former used PCB coil, the observed data scattering is increased resulting in a poor coefficient of determination $\mathrm{R}^{2}=0.82$. As the sensor signal is dominated by the influence of the primary flux, the feedback effect of the secondary flux is hardly detectable. Therefore a linear variable differential transformer is investigated. In this case, the influence of the primary flux is eliminated from the measuring voltage. Furthermore, this setup allows measurements over a broad frequency range in contrast to the sole observation of the resonance frequency as done with the former used setup. Based on theoretical considerations, we could show that the in-phase component of the measuring voltage is proportional to the permittivity of the culture medium and therefore to the cell density in the medium. At a measuring frequency of $300 \mathrm{kHz}$, we could show a linear dependency with a coefficient of determination $\mathrm{R}^{2}=0.98$ between measuring voltage and optical density up to an $\mathrm{OD}=$ 5. Therefore, we conclude that this approach is most promising to realize a measuring system for accurate, continuous and noninvasive monitoring of cell growth in disposable bioreactors. In future work, we will focus on the improvement of our setup, e.g. by realizing an LTCC based linear variable differential transformer. Subsequently, we will test this setup on different types of cells, establish a statistical basis for the determination of accuracy and test the dynamic range of the sensor. Furthermore, it will be investigated if any additional information (e.g. osmolarity) can be determined from the measuring signal by observing different frequency points or the imaginary part of the measuring signal.

\section{References}

[1] V. Singh, Disposable bioreactor for cell culture using wave-induced agitation, Cytotechnology 30 (1-3) (1999) 149-158.

[2] R.A. Rader, E.S. Langer, Upstream single-use bioprocessing systems, BioProcess Int. 10 (2) (2012).

[3] A. Glindkamp, et al., Sensors in disposable bioreactors status and trends, Adv. Biochem. Eng./Biotechnol. 115 (2009) 145-169.

[4] A. Bluma, et al., In-situ imaging sensors for bioprocess monitoring: state of the art, Anal. Bioanal. Chem. 398 (6) (2010) 2429-2438.

[5] S. Beutel, S. Henkel, In situ sensor techniques in modern bioprocess monitoring, Appl. Microbiol. Biotechnol. 91 (6) (2011) 1493-1505.

[6] P. Biechele, C. Busse, D. Solle, T. Scheper, K. Reardon, Sensor systems for bioprocess monitoring, Eng. Life Sci. 15 (5) (2015) 469-488.

[7] R. Eibl, S. Kaiser, R. Lombriser, D. Eibl, Disposable bioreactors: the current state-of-the-art and recommended applications in biotechnology, Appl. Microbiol. Biotechnol. 86 (1) (2010) 41-49.

[8] A.S. Arnoux, L. Preziosi-Belloy, G. Esteban, P. Teissier, C. Ghommidh, Lactic acid bacteria biomass monitoring in highly conductive media by permittivity measurements, Biotechnol. Lett. 27 (20) (2005) 1551-1557.

[9] J.P. Carvell, J.E. Dowd, On-line measurements and control of viable cell density in cell culture manufacturing processes using radio-frequency impedance, Cytotechnology 50 (1) (2006) 35-48.

[10] D.W. Logan, J.P. Carvell, M.P.H. Lee, Creating new opportunities in process control through radio frequency impedance spectroscopy, BMC Proceed. 5 (Suppl 8) (2011).

[11] Recombinant protein production in yeast: Methods and protocols, in: R.M. Bill (Ed.), Humana Press, New York NY u.a, 2012.

[12] S.M. Palmer, E.R.S. Kunji, Online monitoring of biomass accumulation in recombinant yeast cultures, Methods Mol. Biol. (Clifton, N.J.) 866 (2012) 165-179. 
[13] B. Sonnleitner, "Automated Measurement and Monitoring of Bioprocesses: Key Elements of the M3C Strategy," in Advances in Biochemical Engineering/Biotechnology, vol. 132, Measurement, Monitoring, Modelling and Control of Bioprocesses, C.-F. Mandenius and N. J. Titchener-Hooker, Eds., Berlin, Heidelberg: Springer, 2013, pp. 1-33.

[14] B.J. Downey, L.J. Graham, J.F. Breit, N.K. Glutting, A novel approach for using dielectric spectroscopy to predict viable cell volume (VCV) in early process development, Biotechnol. Progress 30 (2) (2014) 479-487.

[15] C. Justice, et al., Process control in cell culture technology using dielectric spectroscopy, Biotechnol. Adv. 29 (4) (2011) 391-401.

[16] A.A. Neves, D.A. Pereira, L.M. Vieira, J.C. Menezes, Real time monitoring biomass concentration in Streptomyces clavuligerus cultivations with industrial media using a capacitance probe, J. Biotechnol. 84 (1) (2001) 45-52.

[17] C. Cannizzaro, R. Gugerli, I. Marison, U. von Stockar, On-line biomass monitoring of CHO perfusion culture with scanning dielectric spectroscopy, Biotechnol. Bioeng. 84 (5) (2003) 597-610.

[18] T. Nacke, A. Barthel, D. Frense, M. Meister, B.P. Cahill, Application of high frequency sensors for contactless monitoring in disposable bioreactors, Chemie Ingenieur Technik 85 (1) (2013) 179-185.

[19] T. Nacke, A. Barthel, B.P. Cahill, M. Meister, Y. Zaikou, High frequency fluidic and microfluidic sensors for contactless dielectric and in vitro cell culture measurement applications, J. Phys.: Conf. Ser. 434 (2013) 12034.

[20] T. Reinecke, P. Biechele, V. Schulte, T. Scheper, S. Zimmermann, Low-cost sensor system for noninvasive monitoring of cell growth in disposable bioreactors, Procedia Eng. 120 (2015) 548-551.

[21] B. Clarke, A Guide to the Characterisation of Dielectic Materials at RF and Microwave Frequencies, The Institute of Measurement and Control, London, 2003.

[22] T. Reinecke, et al., A novel coplanar probe design for fast scanning of edema in human brain tissue via dielectric measurements, Sens. Actuators B: Chem. 220 (2015) 522-527.

[23] A.M. Nicolson, G.F. Ross, Measurement of the intrinsic properties of materials by time-domain techniques, IEEE Trans. Instrum. Meas. 19 (4) (1970) 377-382.

[24] J. Baker-Jarvis, E.J. Vanzura, W.A. Kissick, Improved technique for determining complex permittivity with the transmission/reflection method, IEEE Trans. Microwave Theory Technol. 38 (8) (1990) 10961103.

[25] $\varnothing$. Martinsen, S. Grimnes, H.P. Schwan, Interface phenomena and dielectric properties of biological tissue, in: P. Somasundaran (Ed.), Encyclopedia of Surface and Colloid Science, 2nd ed., Taylor \& Francis, Boca Raton, 2006.

[26] T. Reinecke, P. Biechele, M. Frickhöffer, T. Scheper, S. Zimmermann, Non-invasive online monitoring of cell growth in disposable bioreactors with a planar coil, Procedia Eng. 168 (2016) 582-585.

[27] K. Asami, E. Gheorghiu, T. Yonezawa, Real-time monitoring of yeast cell division by dielectric spectroscopy, Biophys. J. 76 (6) (1999) 3345-3348.

[28] S.A. Siano, Biomass measurement by inductive permittivity, Biotechnol. Bioeng. 55 (2) (1997) 289-304.

[29] T. Meissner, F.J. Wentz, The complex dielectric constant of pure and sea water from microwave satellite observations, IEEE Trans. Geosci. Remote Sens. 42 (9) (2004) 1836-1849.

[30] K.G. Ong, J. Wang, R.S. Singh, L.G. Bachas, C.A. Grimes, Monitoring of bacteria growth using a wireless, remote query resonant-circuit sensor: application to environmental sensing, Biosens. Bioelectron. 5 (2001) 305-312.

[31] Y. Imanaka, Multilayered Low Temperature Cofired Ceramics (LTCC) Technology, Springer, New York NY, 2005.

[32] M. Sobocinski, J. Putaala, H. Jantunen, Multilayer low-temperature co-fired ceramic systems incorporating a thick-film printing process, Printed Films: Materials Sci. Appl. Sensors, Electronics Photonics 134 (2012).

[33] R. Hahn, S. Krumbholz, H. Reichl, Low profile power inductors based on ferromagnetic LTCC technology, in: Proceedings/56th Electronic Components and Technology Conference, 2006: 30 May2 June 2006, [San Diego, CA], Piscataway, NJ: IEEE Operations Center, 2006, pp. 528-533. 
[34] Y.M. Hwang, W.L. Lu, C.T. Pan, Development and fabrication of an LTCC multilayer coil inducer in a vibration-based electromagnetic meso-generator, Microelectron. J. 41 (6) (2010) 338-346.

[35] H.-J. Kim, Y.-J. Kim, J.-R. Kim, An Integrated LTCC Inductor Embedding NiZn Ferrite, IEEE Trans. Magn. 42 (10) (2006) 2840-2842.

[36] C.-Y. Kim, H.-J. Kim, J.-R. Kim, An integrated LTCC inductor, IEEE Trans. Magn. 41 (10) (2005) 35563558.

[37] R.D. Agosti, L. Jouve, H. Greppin, Computer-assisted measurements of plant growth with linear variable differential transformer (LVDT) sensors, Arch. Sci. 50 (3) (1997) 233-244.

[38] W.H. Aellig, A new technique for recording compliance of human hand veins, Br. J. Clin. Pharmacol. 11 (3) (1981) 237-243.

[39] A.D. Woolfson, D.F. McCafferty, S.P. Gorman, P.A. McCarron, J.H. Price, Design of an apparatus incorporating a linear variable differential transformer for the measurement of type III bioadhesion to cervical tissue, Int. J. Pharm. 84 (1) (1992) 69-76.

[40] A.P. Gregory, R.N. Clarke, Tables of the Complex Permittivity of Dielectric Reference Liquids at Frequencies up to $5 \mathrm{GHz}$, NPL, 2001.

\begin{abstract}
Biographies
Tobias Reinecke Tobias Reinecke was born in Hildesheim, Germany, in 1983. He received the Dipl.-Ing. degree in electrical engineering from the Leibniz Universität Hannover, Hannover, Germany in 2011. Since 2011, he has been working as a Research Assistant at the Institute of Electrical Engineering and Measurement Technology at Leibniz Universität Hannover. His current research interests include miniaturized gas sensors, ionization sources for IMS applications and electromagnetic sensors for biomedical applications.
\end{abstract}

Philipp Biechele Philipp Biechele was born in Memmingen, Germany, in 1985. He received the MSc. degree in pharmaceutical biotechnology from the University of Ulm, Ulm, Germany in 2013. Since 2013, he has been working as a Research Assistant at the Institute of Technical Chemistry at Leibniz University Hannover. His current research interests include modern sensor systems for disposable bioreactors, bioprocess engineering and bioprocesse analytics.

Maciej Sobocinski Maciej Sobocinski was born 1983 in Poland. He received the M.Sc. degree in electronics and telecommunication from the Wroclaw University of Techonology, Wroclaw, Poland, in 2007, and the Ph.D. degree in electronic materials and components from the University of Oulu, Oulu, Finland, in 2014. He is currently a post doc at the University of Oulu focusing on Low Temperature Co-fired Ceramic technology for novel applications. Main areas of interest include sensors, actuators, microfluidics and smart packages.

Henning Suhr Henning Suhr was born in Hanover, Germany, in 1991. He started studying at the Leibniz Universität Hannover, Germany, in 2011 and will receive the M.Sc. degree in electrical engineering in 2017. His research interests include sensors and drive technology.

Kai Bakes Kai Niklas Bakes was born in Gehrden, Germany, in 1998. During a volunteer year of science at the Institute of Electrical Engineering and Measurement Technology at Leibniz Universität Hannover, he researched on Ion Mobility Spectrometry, mercury detection and electromagnetic sensors for cell growth observation.

Dörte Solle Dörte Solle was born in Lehrte, Germany, in 1968. She received the exam in biology and chemistry in 1998 and the Dr. rer. nat. in 2003 from the University of Hannover. She worked in the Fraunhofer Institute for Experimental Medicine in Hannover and moved in 2008 to the Institute of Technical Chemistry at Leibniz University of Hannover, Hannover, Germany as senior scientist for spectroscopy and chemometrics in bioprocess technology.

Heli Jantunen ( $\mathrm{M}^{\prime} 02$ ) received the M.Sc. degree in astronomy and physics, the Diploma degree in educa- tion, the M.Sc. degree in electrical engineering, and the Dr.Tech. degree (Hons.) in microelectronics from the University of Oulu, Oulu, Finland, in 1982, 1985, 1989, and 2001, respectively. After 10 years in industry as a Project Manager and Managing Director, she joined the Microelectronics and Materials Physics Laboratories, 
Department of Electrical Engineering, University of Oulu, in 1999. Her research interests include novel sensors and RF applications by implementation of advanced microwave and functional materials, structures and nanotechnology into multifunctional micromodules and printed electronics devices. Dr. Jantunen is a member of the Research Council for Natural Sciences and Engineering, Academy of Finland, the Chair of the Scientific Advisory Board of Finnish Defense (Matine), and Academician of the World Academy of Ceramics (WAC). Currently, she is devoted to her ERC (European Research Council) Advanced Grant project.

Thomas Scheper Thomas Scheper is professor for Technical Chemistry at the University of Hannover. He received his diploma and $\mathrm{PhD}$ degree at the University of Hannover in Chemistry. After a postdoctorate at Caltech (Prof. Jay Bailey) he performed his Habilitation in Technical Chemistry in Hannover. Afterwards, he was professor for biochemistry at the University of Münster. His research interests lie in the design and optimization of bioprocesses (incl. downstreaming). A major focus is the development of bioanalytical systems and the application to industrial processes. These tools help to monitor the intra- and extracellular parameters of the biological systems during cultivation processes. Immunoanalysis is studied analyze complex mixtures of various origin, protein chips are under development.

Stefan Zimmermann Stefan Zimmermann received his Diploma in Electrical Engineering in 1996 and his Dr.Ing. in 2001 from the Technical University Hamburg- Harburg, Germany. In2001, he joined the Berkeley Sensor and Actuator Center, University of California, USA as a postdoctoral scientist with support of a FeodorLynen Fellowship of the Alexander von Humboldt Foundation. In 2004, he joined the Research Unit of Dräger, Germany, where he worked on sensors for medical and safety applications. His latest position was head of Chemical and Biochemical Sensors. In 2009, he joined the Leibniz University Hannover, Germany as a full professor in sensors and measurement technology. 